\title{
The applicability of the ACOSOG Z0011 Criteria to breast cancer patients in Hong Kong
}

\author{
Vivian Man ${ }^{1}$, Man Shan Lo², Ava Kwong ${ }^{1}$ \\ ${ }^{1}$ Division of Breast Surgery, Department of Surgery, Queen Mary Hospital, Hong Kong, China; ${ }^{2}$ Li Ka Shing Faculty of Medicine, The University \\ of Hong Kong, Hong Kong, China \\ Contributions: (I) Conception and design: V Man, MS Lo; (II) Administrative support: V Man, A Kwong; (III) Provision of study materials or patients: \\ V Man, A Kwong; (IV) Collection and assembly of data: V Man, MS Lo; (V) Data analysis and interpretation: V Man; (VI) Manuscript writing: All \\ authors; (VII) Final approval of manuscript: All authors. \\ Correspondence to: Ava Kwong. K1401, Queen Mary Hospital, Hong Kong, China. Email: avakwong@hku.hk.
}

Background: The ACOSOG Z0011 trial demonstrated safe omission of axillary lymph node dissection (ALND) in patients with one or two positive sentinel lymph nodes (SLNs) receiving breast conservative surgery, followed by whole breast irradiation and adjuvant systemic treatment. This study aims to evaluate the exportability of the ACOSOG Z0011 criteria in an Asian/Chinese cohort residing in Hong Kong.

Methods: Retrospective analysis of a prospectively maintained database in a University affiliated tertiary breast centre was performed from June 2014 to May 2019. All breast cancer patients with no palpable adenopathy before surgery, one or more positive sentinel lymph nodes on histological examination and no prior neoadjuvant systemic treatment were recruited. Patients were grouped as eligible or ineligible according to the ACOSOG Z0011 criteria. The eligible group was compared with the sentinel alone group in the ACOSOG Z0011 cohort.

Results: Two hundred and forty-eight patients were recruited into the study. Sixty patients (24\%) met the ACOSOG Z0011 criteria and could potentially avoid ALND. A higher percentage of clinical T2 tumors were observed in our eligible group than in the ACOSOG Z0011 trial $(\mathrm{P}=0.002)$. The histological subtype, tumor grade, estrogen receptor (ER)/progestogen receptor (PR) status and lymphovascular invasion status did not differ. There was no statistically significant difference in the proportion of SLN micrometastasis and macrometastasis between the two groups.

Conclusions: This study demonstrated clinical similarities between our eligible cohort and the ACOSOG Z0011 cohort, which confirms exportability of the ACOSOG Z0011 criteria to a subset of population in Hong Kong.

Keywords: Breast cancer; biopsy; sentinel lymph node (SLN); lymph node dissection

Submitted Nov 29, 2020. Accepted for publication Mar 25, 2021.

doi: $10.21037 /$ cco-20-239

View this article at: http://dx.doi.org/10.21037/cco-20-239

\section{Introduction}

The optimal axillary management in breast cancer patients continues to evolve in the past few decades. Axillary lymph node dissection (ALND), which was once the standard of care in breast cancer surgery, now becomes an over-treatment in a significant proportion of breast cancer patients. In patients with clinically node-negative breast cancer, sentinel lymph node biopsy (SLNB) has replaced ALND as the gold standard for axillary staging and loco-regional control $(1,2)$. Results from the phase 3

\footnotetext{
^ ORCID: 0000-0001-8306-4904.
} 
International Breast Cancer Study Group (IBCSG) 23-01 randomized controlled trials $(3,4)$ further reinforced the role of SLNB alone in patients with sentinel lymph node (SLN) micrometastases. Together with the publication from the American College of Surgeons Oncology Group Z0011 (ACOSOG Z0011) randomized clinical trials $(5,6)$, breast cancer patients with minimal SLN involvement may now be spared a completion ALND without compromising a 10-year oncological outcome (6). Consequently, an updated clinical practice guideline of omitting completion ALND in this subgroup of patient population is recommended by the American Society of Clinical Oncology (ASCO) (7) and the National Comprehensive Cancer Network (NCCN) (8). It is practice changing and there have been a significant decline in the rate of ALND worldwide $(9,10)$.

The ACOSOG Z0011 study is a seminal study and yet the only randomized controlled trial including patients with SLN macrometastases. It has been challenged in several ways. The excellent local and distant outcomes in the Z0011 study group, enrollment not meeting the accrual goal, slight inequalities in several prognostic characteristics and a significant number of radiotherapy protocol deviations have raised concerns from the National Institute for Health and Care Excellence (NICE) (11) in changing their recommendation on axillary treatment. Completion axillary dissection is still the common practice in patients with positive sentinel lymph nodes in Hong Kong. This study aims to review the clinical characteristics of our breast cancer patients and to evaluate the exportability of Z0011 criteria in our patient population. We present the following article in accordance with the STROBE reporting checklist (available at http://dx.doi.org/10.21037/cco-20-239).

\section{Methods}

\section{Patient recruitment}

The ACOSOG Z0011 approach was adopted in our institution since June 2019. We conducted a retrospective analysis from a prospectively maintained database in a University affiliated tertiary breast cancer centre in Hong Kong from June 2014 to May 2019. This retrospective study was approved by the Ethical Committee of the University of Hong Kong/ Hospital Authority (HKU/ HA HKW UW 09-045). It followed the principles of the Declaration of Helsinki (as revised in 2013) and informed consents were obtained from patients who were willing to participate. Patients were recruited if they fulfilled the following criteria: no palpable adenopathy before surgery, one or more positive SLNs on histological examination, and no prior neoadjuvant systemic treatment. As per the ACOSOG Z0011 trial, patients who had SLNs positive only after immunohistochemical staining were excluded. The recruited patients were then divided into two groups according to the Z0011 trial criteria, namely women with clinical $\mathrm{T} 1$ or $\mathrm{T} 2$ invasive breast cancer, no palpable axillary adenopathy, one or two SLNs containing metastases and had planned lumpectomy, tangential whole-breast irradiation and adjuvant systemic treatment. Patients who met the Z0011 criteria were grouped under the eligible group, while those beyond criteria were grouped as ineligible.

\section{Clinical management and pathological assessment}

In our breast cancer centre, all patients received triple assessment to establish the diagnosis of breast cancer. Both mammogram and ultrasound of breasts and axillae were routinely done to assess the pre-treatment clinical $\mathrm{T}$ and $\mathrm{N}$ staging. All patients with clinically node-negative invasive breast cancer received SLNB to complete the axillary staging. SLN localization were performed with radioisotope with or without patent blue dye according to surgeon's preference. Non-SLN were defined as suspicious nodes that were neither hot nor blue during SLNB or nodes that were removed during completion ALND. Intraoperative frozen section of SLNs or suspicious non-SLNs and subsequent routine haematoxylin and eosin (H\&E) stain were done in all included patients. Immunohistochemistry was not routinely performed unless there was suspicion of nodal metastasis. Based on a protocal prior to the era of Z0011 study, immediate completion ALND was carried out if any of the SLNs or non-SLNs was positive on frozen section analysis. If SLN metastasis was detected in paraffin section only, a delayed completion ALND was carried out in a separate session. Pathology of SLNs was reported as normal, or containing macrometastases $(>2 \mathrm{~mm})$, micrometastases $(>0.2 \mathrm{~mm}, \leq 2 \mathrm{~mm})$ or isolated tumor cells $(\leq 0.2 \mathrm{~mm})$. For patients undergoing breast conservative surgery, 'no ink on tumor' was regarded as an adequate resection margin (12) or else a second operation was performed to ensure a clear resection margin. Histological grade was reported according to the modified Scarff-Bloom-Richardson system. All pathological results were discussed in our multidisciplinary meeting and adjuvant treatment was given at the discretion of breast oncology specialists. 


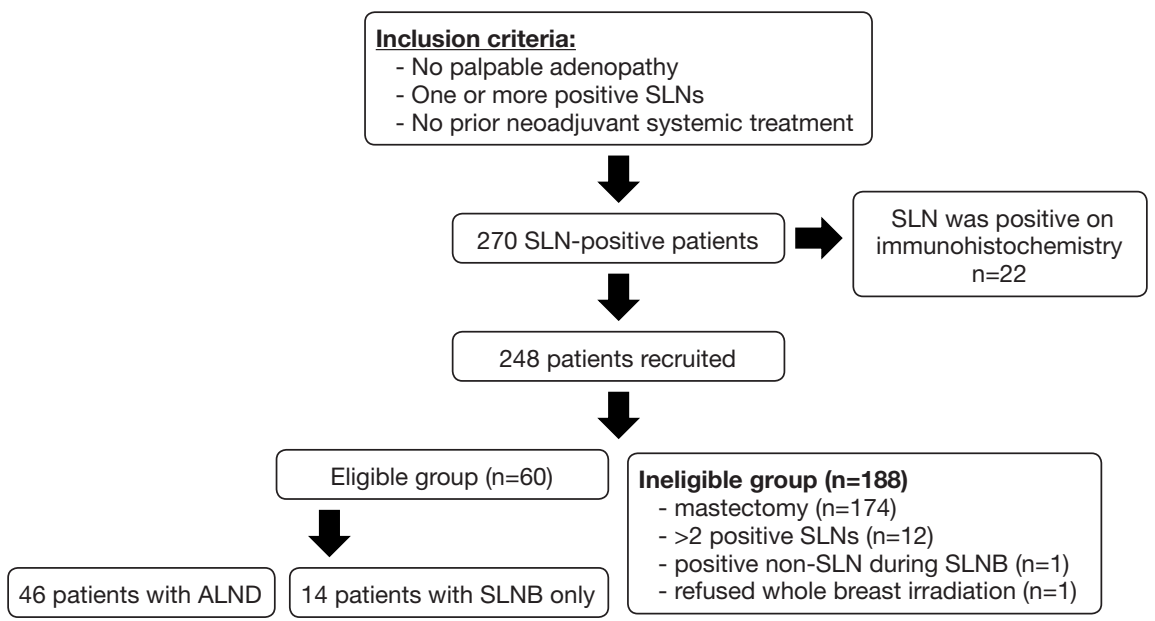

Figure 1 Breast cancer patients with positive SLNs from June 2014 to May 2019. SLN, sentinel lymph node; ALND, axillary lymph node dissection.

\section{Statistical analysis}

Information on patients' demographics and tumor characteristics were collected and tabulated in percentages. Comparison was made between the eligible group of our cohort and the sentinel alone arm in the Z0011 cohort ( $n=436$, in intention to treat). Missing data was tabulated and managed by listwise deletion. All statistical analyses were performed with IBM SPSS, Statistic 24. Chi-square test was used for the following categorical variables: patient's age group, clinical T staging, Estrogen receptor (ER)/ progestogen receptor (PR) status, histological subtype, tumor grade and presence of lymphovascular invasion. A P value of $<0.05$ was considered significant.

\section{Results}

From June 2014 to May 2019, 1249 breast cancer patients received SLNB in our institution and 270 patients (21.6\%) with one or more positive SLNs were identified from a prospectively maintained clinical database. 22 patients were excluded because the SLNs were only positive after immunohistochemistry, 5 of them had micrometastatic SLNs and 17 had isolated tumor cells. A total of 248 patients were included in the analysis (Figure 1).

Mean patient age was 52 years (range, 24-85 years) and mean tumor size was $22.9 \mathrm{~mm}$ (range, 2-115 mm). 60 patients (24\%) who met the Z0011 criteria for omitting ALND were included in the eligible group and 188 patients $(76 \%)$ who did not meet the criteria were grouped into the ineligible group. Within the ineligible group, 174 patients received mastectomy, 12 patients had $>2$ positive SLNs, 1 patient had positive non-SLN during SLNB and 1 patient refused adjuvant whole breast irradiation.

Patients' demographics and tumor characteristics of our eligible group and the sentinel alone arm in the ACOSOG Z0011 trial are demonstrated in Table 1. A higher percentage of clinical T2 tumors were observed in our eligible group than in the sentinel alone arm of Z0011 trial $(50 \%$ vs. $29.4 \% ; \mathrm{P}=0.002)$. Otherwise there were no statistically significant difference between these two cohorts in terms of age, histological subtype, tumor grade, ER/ PR status and lymphovascular permeation status. The proportion of SLN micrometastases (36.7\% vs. $44.8 \%$ ) and macrometastases $(61.7 \%$ vs. $55.2 \%)$ did not differ $(\mathrm{P}=0.35)$.

Within our eligible group, forty-six out of sixty patients (76.7\%) received completion ALND. Thirty-eight of them had one positive SLN and eight patients had two. Among these forty-six patients, nine patients (19.6\%) had additional metastatic non-SLNs (range, 1-8) in the ALND specimen. Four of them had macrometastatic non-SLNs and two had micrometastatic non-SLNs. There was missing information concerning the size of invasive focus in the remaining three patients. All these 9 patients had macro-metastatic SLNs and five of them had clinical T2 disease. Fourteen out of the sixty patients (23.3\%) in the eligible cohort received SLNB only and 11 of them had either micrometastatic SLN or isolated tumor cells.

All patients in the eligible group received whole breast irradiation and 37 patients $(61.7 \%)$ received additional 
Table 1 Clinical characteristics of patients of the eligible group in current study and sentinel group in the ACOSOG Z0011 trial

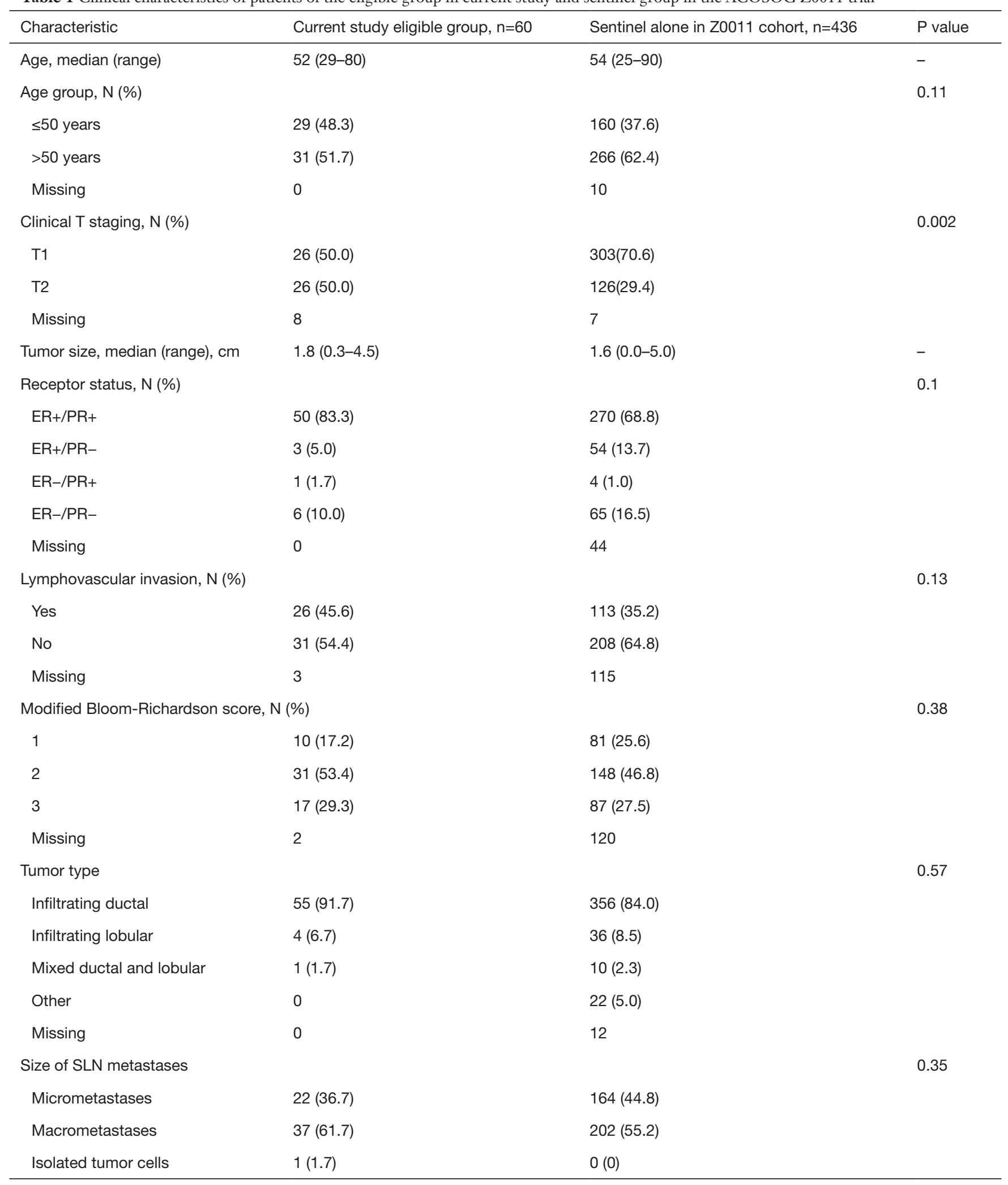


third field nodal irradiation. 45 patients received adjuvant chemotherapy and 54 patients was put on adjuvant hormonal treatment. The median follow-up time was 45 months (range, 13-73 months). There was no axillary recurrence during the follow-up period. Three patients developed distant recurrence and one of them passed away in 39 months. One patient experienced ipsilateral breast recurrence in 30 months and received completion mastectomy.

\section{Discussion}

The ACOSOG Z0011 trial $(5,6)$ is the largest prospective randomized trial to evaluate the role of completion ALND in SLN macrometastases. It defined a group of eligible patients with 1 or 2 SLN metastases receiving breast conservative surgery, whole-breast irradiation and adjuvant systemic treatment to safe omission of completion ALND. The equivalent loco-regional recurrence and survival rates in the sentinel alone group have resulted in a paradigm shift in axillary management worldwide. Fillion et al. (10) identified 800 patients who received breast conserving treatment from 2007 to 2013 and found a significant reduction in completion ALND from $78 \%$ in the pre-Z0011 era to $21 \%$ in their post-Z0011 cohort. Similarly, the MD Anderson group (13) has shown a marked decrease in ALND from $85 \%$ to $25 \%$ after Z0011 and this trend has become more pronounced over time. Similar trend of ALND reduction of up to $34 \%$ have likewise been observed in Europe (14,15) and Australia (16). This abrupt transition away from ALND is in no doubt associated with less surgical morbidities (4,17). In the ACOSOG Z0011 trial, the sentinel alone group was found to have less wound infection, axillary seromas, axillary parasthesia and reported lymphedema (18). Bhatt et al. (19) also noticed a reduction in lymphedema new referrals by $20 \%$ after implementation of Z0011. The ACOSOG Z0011 study has thus been described as a 'practice-changing trial' in the States (20).

Despite all the encouraging results, arguments have been raised regarding this non-ALND approach in SLN metastases. The ACOSOG Z0011 trial is the only one trial evaluating SLN macrometastases with a moderate risk of bias (21). It has been criticized by the enrollment of only patients with a good prognosis (22) and inequalities in prognostic factors between the study groups (21). Therefore questions have been raised on the exportability of results to other patient populations. Camp et al. (9) retrospectively reviewed their data at Massachusetts General Hospital in 2009 and observed a $72 \%$ eligibility in their patient cohort with potential benefit in peri-operative cost savings. Delpech et al. (23) established similarities of clinical characteristics in their French cohort with the Z0011 group and found almost $70 \%$ of their patient population were eligible for omitting ALND. In the large multicenter retrospective population based study by Verheuvel et al. (24), the clinical characteristics were also comparable and nearly $61 \%$ of the SLN-positive patients potentially fulfilled the Z0011 criteria.

However this high exportability within the western population is not observed in the current study. In Hong Kong, only $24 \%$ of our patient population were found eligible for Z0011 criteria. This is consistent with the findings from Liu et al. (25), who found $23 \%$ of their patients eligible for ALND omission in the Chinese population. This difference in the applicability of Z0011 criteria probably reflects some differences in the clinical characteristics between the Asian and Western population. In our study, $70 \%$ of our patient population received mastectomy and there was a higher proportion of clinical T2 tumors in the eligible group when compared with the Z0011 cohort. This could be linked to the lack of nationwide mammographic screening in Hong Kong, in which more than $80 \%$ of first breast cancer detection was due to symptomatic presentation and majority as painless lump (26). Both symptomatic presentation and T2 tumors have been shown in local population-wide study to be inversely associated with the rate of breast conservative treatment in Hong Kong population (27). In addition, the relatively small breast size of Chinese women (28) and a more conservative cultural attitudes $(27,28)$ were accountable for the high mastectomy rate in Hong Kong. The Korean group (29) also found larger tumors in their Asian Z0011-eligible patients which are comparable with our study group. In addition, they had higher-grade tumors and more frequent lymphovascular permeation in their cohort. Similarly, a recent prospective study by the Japanese group (30) found a higher frequency of tumors with lymphovascular permeation and SLN macrometastases in their Z0011-eligible cohort. Nevertheless, despite these differences, both the Korean (29) and Japanese (30) demonstrated safe application of Z0011 trial in Asia, with low incidence of disease recurrence.

Another suggested concern from the ACOSOG Z0011 trial is about the under-treatment of patients with extensive nodal disease. Previous studies have shown the risk of nonSLN metastases were up to $26 \%$ in SLN micrometastases 
and up to $63 \%$ in SLN macrometastases $(31,32)$. Several nomograms (33-35) have been developed and validated in the pre-Z0011 era to predict the risk of non-SLN metastases and need for completion ALND. For instance, in our study, $60 \%$ of our Z0011-eligible patients had SLN macrometastases and around $20 \%$ had additional metastatic non-SLNs. This is indeed much lower than reported in the original Z0011 [5-6] and several exportability trials worldwide $(23,25,36)$. In view of the very low recurrence rate described in the ACOSOG Z0011 trial [5-6], this application of nomograms in ALND decision appears to become obsolete now. In the presence of whole breast irradiation and adjuvant systemic treatment, probably not all non-SLN metastases develop into clinical detectable disease. One criticism of the ACOSOG Z0011 trial is the significant radiotherapy protocol deviations in the study. An independent analysis (37) of radiation fields in a subset of participants in the ACOSOG Z0011 trial identified 18.9\% protocol-prohibited nodal field irradiation, including $15 \%$ to the supraclavicular region. This non-ALND approach described in the ACOSOG Z0011, was in contrast to the AMAROS trial (38), which randomized patients with clinical T1 or T2 invasive breast cancer and positive SLN to ALND or axillary radiotherapy. Axillary radiotherapy included the contents of all three levels of the axilla and the medial part of the supraclavicular fossa and this was prohibited in the ACOSOG Z0011 protocol. Together with the favourable results of axillary radiotherapy in the AMAROS trial (38), the optimal adjuvant radiotherapy protocol in this group of patients is yet to be determined. In order to address these uncertainties, several ongoing studies, for example POSNOC (39), BOOG 2013-07 (40), SINODAR ONE (41) and SENOMAC trial (42), are trying to evaluate the oncological safety in forgoing ALND or axillary radiotherapy in patients with limited spread to SLNs, especially in patients receiving mastectomy.

This study is limited by its retrospective nature with probable patient selection bias and recall bias. There are inevitably some missing information in the initial histopathological report and our database. In addition, there are limited data from the Z0011 trial with respect to the human epidermal growth factor 2 (HER2) status and nodal extracapsular extension. However we believe it reflects the genuine difference in clinical characteristics between the our cohort which are Chinese and the reported cohort which are mainly Westerners. Our patient population generally presents with larger tumors and we have a higher rate of mastectomy in Hong Kong. All these contribute to the lower proportion of patients eligible for omitting ALND.

In conclusion, this study demonstrated a lower exportability of the ACOSOG Z0011 criteria to the Chinese patients in Hong Kong. While the Z0011 trial is to be adopted as our standard practice, we believe a standardization of adjuvant radiotherapy is also imminent for the good results. In addition, in view of high mastectomy rate in Hong Kong, additional work remains to be done to better define other low-risk patient population for whom completion ALND can be avoided. On the other hand, the extension of indication of breast conservative surgery with adoption of oncoplastic techniques should be sought to allow more widespread application of Z0011.

\section{Acknowledgments}

The abstract has been accepted for poster presentation in the RCSEd/CSHK Conjoint Virtual Scientific Congress 2020 and in the journal Surgical Practice.

Funding: None.

\section{Footnote}

Reporting Checklist: The authors have completed the STROBE reporting checklist. Available at http://dx.doi. org/10.21037/cco-20-239

Data Sharing Statement: Available at http://dx.doi. org/10.21037/cco-20-239

Conflicts of Interest: All authors have completed the ICMJE uniform disclosure form (available at http://dx.doi. org/10.21037/cco-20-239). The authors have no conflicts of interest to declare.

Ethical Statement: The authors are accountable for all aspects of the work in ensuring that questions related to the accuracy or integrity of any part of the work are appropriately investigated and resolved. The study was conducted in accordance with the Declaration of Helsinki (as revised in 2013). The study was approved by the Ethical Committee of the University of Hong Kong/Hospital Authority (HKU/HA HKW UW 09-045) and informed consent was taken from all the patients.

Open Access Statement: This is an Open Access article distributed in accordance with the Creative Commons 
Attribution-NonCommercial-NoDerivs 4.0 International License (CC BY-NC-ND 4.0), which permits the noncommercial replication and distribution of the article with the strict proviso that no changes or edits are made and the original work is properly cited (including links to both the formal publication through the relevant DOI and the license). See: https://creativecommons.org/licenses/by-nc-nd/4.0/.

\section{References}

1. Krag DN, Anderson SJ, Julian TB, et al. Sentinel-lymphnode resection compared with conventional axillarylymph-node dissection in clinically node-negative patients with breast cancer: overall survival findings from the NSABP B-32 randomised phase 3 trial. Lancet Oncol 2010;11:927-33.

2. Veronesi U, Viale G, Paganelli G, et al. Sentinel lymph node biopsy in breast cancer: ten-year results of a randomized controlled study. Ann Surg 2010;251:595-600.

3. Galimberti V, Cole BF, Zurrida S, et al; International Breast Cancer Study Group Trial 23-01 investigators. Axillary dissection versus no axillary dissection in patients with sentinel-node micrometastases (IBCSG 23-01): a phase 3 randomised controlled trial. Lancet Oncol 2013;14:297-305. Erratum in: Lancet Oncol 2013;14:e254.

4. Galimberti V, Cole BF, Viale G, et al; International Breast Cancer Study Group Trial 23-01. Axillary dissection versus no axillary dissection in patients with breast cancer and sentinel-node micrometastases (IBCSG 23-01): 10-year follow-up of a randomised, controlled phase 3 trial. Lancet Oncol 2018;19:1385-93.

5. Giuliano AE, Hunt KK, Ballman KV, et al. Axillary dissection vs no axillary dissection in women with invasive breast cancer and sentinel node metastasis: a randomized clinical trial. JAMA 2011;305:569-75.

6. Giuliano AE, Ballman KV, McCall L, et al. Effect of Axillary Dissection vs No Axillary Dissection on 10-Year Overall Survival Among Women With Invasive Breast Cancer and Sentinel Node Metastasis: The ACOSOG Z0011 (Alliance) Randomized Clinical Trial. JAMA 2017;318:918-26.

7. Lyman GH, Temin S, Edge SB, et al; American Society of Clinical Oncology Clinical Practice. Sentinel lymph node biopsy for patients with early-stage breast cancer: American Society of Clinical Oncology clinical practice guideline update. J Clin Oncol 2014;32:1365-83.

8. Gradishar WJ, Anderson BO, Balassanian R, et al. NCCN Guidelines Insights: Breast Cancer, Version 1.2017. J Natl Compr Canc Netw 2017;15:433-51.
9. Camp MS, Greenup RA, Taghian A, et al. Application of ACOSOG Z0011 criteria reduces perioperative costs. Ann Surg Oncol 2013;20:836-41.

10. Fillion MM, Glass KE, Hayek J, et al. Healthcare Costs Reduced After Incorporating the Results of the American College of Surgeons Oncology Group Z0011 Trial into Clinical Practice. Breast J 2017;23:275-81.

11. National Guideline Alliance (UK). Early and locally advanced breast cancer: diagnosis and management. London: National Institute for Health and Care Excellence (UK); 2018.

12. Moran MS, Schnitt SJ, Giuliano AE, et al. Society of Surgical Oncology-American Society for Radiation Oncology consensus guideline on margins for breast-conserving surgery with whole-breast irradiation in stages I and II invasive breast cancer. Ann Surg Oncol 2014;21:704-16.

13. Caudle AS, Hunt KK, Tucker SL, et al. American College of Surgeons Oncology Group (ACOSOG) Z0011: impact on surgeon practice patterns. Ann Surg Oncol 2012;19:3144-51.

14. Joyce DP, Lowery AJ, McGrath-Soo LB, et al. Management of the axilla: has Z0011 had an impact? Ir J Med Sci 2016;185:145-9.

15. Morigi C, Peradze N, Galimberti V, et al. Feasibility and surgical impact of Z0011 trial criteria in a singleInstitution practice. Breast J 2020;26:1330-6.

16. Ngui NK, Hitos K, Hughes TMD. Effect of the American College of Surgeons Oncology Group Z0011 trial on axillary management in breast cancer patients in the Australian setting. Breast J 2019;25:853-8.

17. Mansel RE, Fallowfield L, Kissin M, et al. Randomized multicenter trial of sentinel node biopsy versus standard axillary treatment in operable breast cancer: the ALMANAC Trial. J Natl Cancer Inst 2006;98:599-609. Erratum in: J Natl Cancer Inst 2006;98:876.

18. Lucci A, McCall LM, Beitsch PD, et al; American College of Surgeons Oncology Group. Surgical complications associated with sentinel lymph node dissection (SLND) plus axillary lymph node dissection compared with SLND alone in the American College of Surgeons Oncology Group Trial Z0011. J Clin Oncol 2007;25:3657-63.

19. Bhatt NR, Boland MR, McGovern R, et al. Upper limb lymphedema in breast cancer patients in the era of Z0011, sentinel lymph node biopsy and breast conservation. Ir J Med Sci 2018;187:327-31.

20. Caudle AS, Hunt KK, Kuerer HM, et al. Multidisciplinary considerations in the implementation of the findings from the American College of Surgeons Oncology Group 
(ACOSOG) Z0011 study: a practice-changing trial. Ann Surg Oncol 2011;18:2407-12.

21. Huang TW, Kuo KN, Chen KH, et al. Recommendation for axillary lymph node dissection in women with early breast cancer and sentinel node metastasis: A systematic review and meta-analysis of randomized controlled trials using the GRADE system. Int J Surg 2016;34:73-80.

22. Jatoi I. Axillary vs sentinel lymph node dissection for invasive breast cancer. JAMA 2011;305:2288; author reply 2290-1.

23. Delpech Y, Bricou A, Lousquy R, et al. The exportability of the ACOSOG Z0011 criteria for omitting axillary lymph node dissection after positive sentinel lymph node biopsy findings: a multicenter study. Ann Surg Oncol 2013;20:2556-61.

24. Verheuvel NC, Voogd AC, Tjan-Heijnen VC, et al. Potential impact of application of Z0011 derived criteria to omit axillary lymph node dissection in node positive breast cancer patients. Eur J Surg Oncol 2016;42:1162-8.

25. Liu M, Wang S, Cui S, et al. The feasibility of the ACOSOG Z0011 Criteria to Chinese Breast Cancer Patients: A Multicenter Study. Sci Rep 2015;5:15241.

26. Hong Kong Breast Cancer Registry Report No. 11 (Issue 2019). Hong Kong Breast Cancer Foundation Web site. Available online: http://www.hkbcf.org [accessed 17.07.20]

27. Chan SWW, Cheung C, Chan A, et al. Surgical options for Chinese patients with early invasive breast cancer: Data from the Hong Kong Breast Cancer Registry. Asian J Surg 2017;40:444-52.

28. Sinnadurai S, Kwong A, Hartman M, e. Breast-conserving surgery versus mastectomy in young women with breast cancer in Asian settings. BJS Open 2018;3:48-55.

29. Jung J, Han W, Lee ES, et al. Retrospectively validating the results of the ACOSOG Z0011 trial in a large Asian Z0011-eligible cohort. Breast Cancer Res Treat 2019;175:203-15.

30. Kittaka N, Tokui R, Ota C, et al. A prospective feasibility study applying the ACOSOG Z0011 criteria to Japanese patients with early breast cancer undergoing breastconserving surgery. Int J Clin Oncol 2018;23:860-6.

31. Chu KU, Turner RR, Hansen NM, et al. Do all patients with sentinel node metastasis from breast carcinoma need complete axillary node dissection? Ann Surg 1999;229:536-41.

32. Turner RR, Chu KU, Qi K, et al. Pathologic features associated with nonsentinel lymph node metastases in patients with metastatic breast carcinoma in a sentinel lymph node. Cancer 2000;89:574-81.
33. Van Zee KJ, Manasseh DM, Bevilacqua JL, et al. A nomogram for predicting the likelihood of additional nodal metastases in breast cancer patients with a positive sentinel node biopsy. Ann Surg Oncol 2003;10:1140-51.

34. Pal A, Provenzano E, Duffy SW, et al. A model for predicting non-sentinel lymph node metastatic disease when the sentinel lymph node is positive. Br J Surg 2008;95:302-9.

35. Kohrt HE, Olshen RA, Bermas HR, et al. Bay Area SLN Study. New models and online calculator for predicting non-sentinel lymph node status in sentinel lymph node positive breast cancer patients. BMC Cancer 2008;8:66.

36. Ngui NK, Elder EE, Jayasinghe UW, et al. Relevance of the American College of Surgeons Oncology Group Z0011 Trial to breast cancer in the Australian setting. ANZ J Surg 2013;83:924-8.

37. Jagsi R, Chadha M, Moni J, et al. Radiation field design in the ACOSOG Z0011 (Alliance) Trial. J Clin Oncol 2014;32:3600-6.

38. Donker M, van Tienhoven G, Straver ME, et al. Radiotherapy or surgery of the axilla after a positive sentinel node in breast cancer (EORTC 10981-22023 AMAROS): a randomised, multicentre, open-label, phase 3 non-inferiority trial. Lancet Oncol 2014;15:1303-10.

39. Goyal A, Dodwell D. POSNOC: A Randomised Trial Looking at Axillary Treatment in Women with One or Two Sentinel Nodes with Macrometastases. Clin Oncol (R Coll Radiol) 2015;27:692-5.

40. van Roozendaal LM, de Wilt JH, van Dalen T, et al. The value of completion axillary treatment in sentinel node positive breast cancer patients undergoing a mastectomy: a Dutch randomized controlled multicentre trial (BOOG 2013-07). BMC Cancer 2015;15:610.

41. Tinterri C, Canavese G, Bruzzi P, et al. SINODAR ONE, an ongoing randomized clinical trial to assess the role of axillary surgery in breast cancer patients with one or two macrometastatic sentinel nodes. Breast 2016;30:197-200.

42. de Boniface J, Frisell J, Andersson Y, et al; SENOMAC Trialists' Group. Survival and axillary recurrence following sentinel node-positive breast cancer without completion axillary lymph node dissection: the randomized controlled SENOMAC trial. BMC Cancer 2017;17:379.

Cite this article as: Man V, Lo MS, Kwong A. The applicability of the ACOSOG Z0011 Criteria to breast cancer patients in Hong Kong. Chin Clin Oncol 2021;10(3):27. doi: 10.21037/cco-20-239 